\title{
A ZigBee wireless sensor network compliant with the IEEE1451 standard
}

\author{
Jorge Higuera, Jose Polo, Manel Gasulla \\ Instrumentation, Sensors and Interfaces Group \\ Universitat Politécnica de Catalunya \\ Castelldefels (Barcelona), Spain \\ higuera@eel.upc.edu,jose.polo@upc.edu, manel.gasulla@upc.edu.
}

\begin{abstract}
Wireless sensor networks are becoming very attractive for monitoring and control applications with smart sensor nodes. We present an implementation of a ZigBee wireless sensor network compliant with the IEEE1451 standard to improve the interoperability. This paper describes the message structure, commands and transactions. Furthermore, a proposal is presented to enhance the IEEE1451.5 ZigBee physical transducer electronic datasheet (PHY TEDS).
\end{abstract}

Keywords: IEEE1451, interoperability, NCAP, WTIM, TEDS, ZigBee, WSN.

\section{INTRODUCTION}

Wireless Sensor Networks (WSNs) are becoming very attractive in many pervasive computing environments and constitute a key technology for monitoring and control. They are composed of low cost, small size and low power nodes. One of the nodes actuates as the coordinator of the network.

The implementation of commercial WSNs involves, in many cases, proprietary application programming interfaces (APIs) and protocols. The use of different proprietary data formats and protocols constitutes a bottleneck for the expansion of these networks. The recent introduction of the IEEE802.15.4 -2006 standard [1] and ZigBee specification can help in adopting a common manufacturer-independent communication protocol for WSNs. At a higher level, the IEEE1451 standard family [2] was introduced in the last decade as a set of open standards, organized around a set of common architectures and protocols to permit the interoperability of multiple smart sensors. In this way, smart sensors can be connected in a plug and play way in a distributed wireless or wired network. The main advantages of this envision is a new design of smart sensors with digital communication standard interface and many options including self diagnosis, autocalibration, multi sensing, data storage in a electronic data sheet, and signal output in engineering units.

The IEEE1451.5 is compatible with radio-specific protocols for WSNs such as 802.11 (WiFi), 802.15.1 (Bluetooth), ZigBee, and 6LowPAN [3]. Still, few implementations have been reported. Sweetser et al. [4] implemented a smart sensor based on IEEE1451 and Bluetooth communication interface. Song and Lee [5] used the IEEE802.11 interface. The same authors [6] provide a set of guidelines for implementing applications based on the IEEE1451 using the unified modeling language (UML). Wobschall [7] shows a wireless smart sensor with point-to-point connectivity by using the IEEE 1451.4 physical layer. However, to the best of our knowledge, no solution has been proposed for an IEEE1451.5 Zigbee network. This paper proposes a Zigbee network compliant with the IEEE1451 standard.

\section{PROPOSED ARCHITECTURE}

Figure 1 shows the basic architecture of a smart IEEE1451 transducer particularized for a wireless sensor based on IEEE1451.5. It includes the following parts:

- Wireless Transducer Interface Module (WTIM). In our architecture, it includes the physical sensors, signal conditioning, a MSP430 microcontroller unit with internal analog to digital converter, and the Transducer Electronic Data Sheet (TEDS) embedded in external serial Flash memory. The TEDS contains information about calibration, functionality parameters, vendor, data units, etc. A WSN is composed of multiple WTIMs

- Network Capability Application Processor (NCAP). The NCAP consists of a processor with an embedded operating system and timing capability. It actuates as the coordinator of the WSN. The NCAP is a device between the wireless transducer modules and the wired network and performs the connections to multiple WTIMs with the same physical layer. In our application the physical layer is IEEE802.15.4. The NCAP may initiate the discovery and association of wireless node at any time.

- Transducer Independent Interface (TII). The IEEE 1451 defines a set of standard interfaces that support different networks: wired and wireless networks. Our approach uses a ZigBee IEEE1451.5 interface.

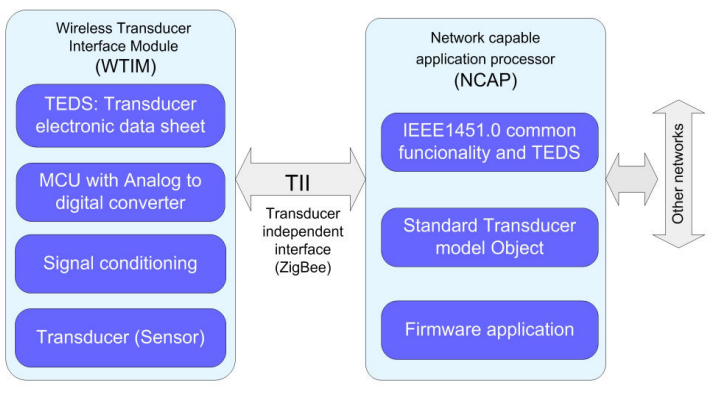

Fig 1. IEEE1451 Smart sensor model. 


\section{A. Network architecture}

Figure 2 shows the proposed WSN architecture with WTIMs and the NCAP. Database support and monitorization via Internet has been added. NCAP and STIMs nodes are implemented with Sky Tmote modules (MoteIV, now Sentilla) [8] working under a real time operative system (RTOS) TinyOS [9]. All firmware for WTIMs and NCAP was developed in NesC language [10].

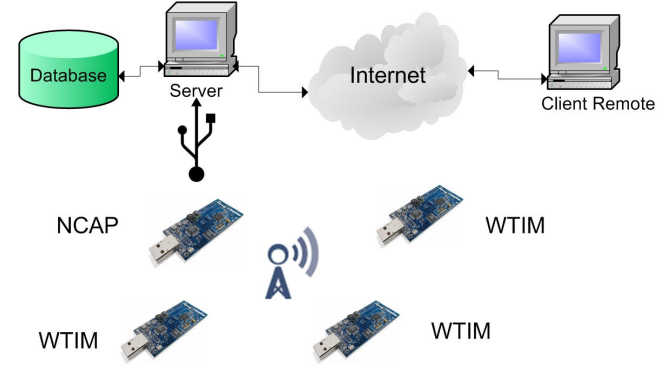

Fig 2. Proposed WSN architecture.

\section{B. Nodes}

Each Sky Tmote module includes several sensors, a 16 bit MSP430 microcontroller, 6 analog input channels, and a Zigbee compliant CC2420 transceiver [11] working at the 2.4 $\mathrm{GHz}$ ISM band. Additionally, a USB interface is present. Figure 3 shows the block diagram of the module.

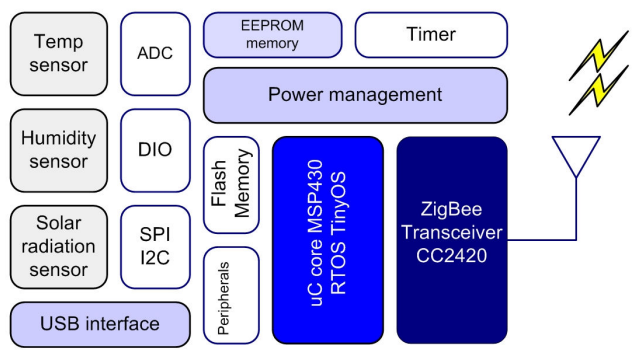

Fig 3. Block diagram of a Sky Tmote module.

\section{NCAP}

The NCAP is composed of a Sky Tmote module connected via USB to a PC. The NCAP coordinates the activity of all WTIMs by sending IEEE1451.0 standard commands through the Zigbee transceiver. Received data from WTIMs are stored in a MySQL database and accessed via Internet.

\section{WTIM}

Each WTIM includes four sensor channels (temperature, humidity, solar radiation and battery voltage), and all mandatory TEDSs stored in an EEPROM memory. Mandatory TEDS include: Meta-TEDS (IEEE1451.0), Transducer Channel TEDS (IEEE1451.0), User's Transducer Name TEDS (IEEE1451.0) and PHY TEDS (IEEE1451.5) for the ZigBee interface. Power is provided by two AA primary batteries of $2700 \mathrm{mAh}$ connected in series.
Firmware has been developed for the WTIMs to read the transducer channels and mandatory TEDS.

\section{E. Power management}

The WTIMs use different operation power modes including active, idle and sleep, in order to preserve power and to extend the battery life. Our application includes a synchronized protocol (SP) for low power operation [12], which establishes and maintains a schedule whereby the entire network wakes up together and then returns to sleep. The activation period is $400 \mathrm{~s}$ and the duty cycle is $1 \%$, so each WTIM only operates for $4 \mathrm{~s}$ in active mode and then returns to sleep mode for $396 \mathrm{~s}$.

\section{F. User interface}

A graphical user interface (GUI) has been developed in LabVIEW that includes the commands compliant with the IEEE1451 ZigBee radio sub-specification to send and receive the information in the WSN and shows all active messages in the frontal panel. Figure 4 shows the command menu of the frontal panel. This application operates in a PC server and users can control and monitor all parameters of the WTIMs channels using the IEEE1451 standard commands.

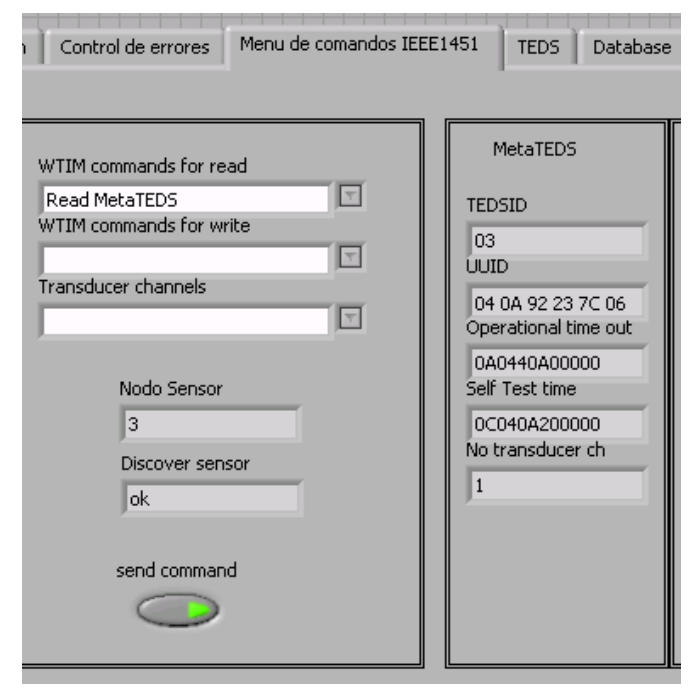

Fig 4 Frontal panel (command menu) of graphical user interface (GUI) implemented.

\section{IEEE1451 TRANSACTIONS, COMMANDS AND TEDS}

IEEE1451.0 Transactions can be initiated either by the NCAP or the WTIM [13]. In our case only transactions initiated by the NCAP have been implemented. Figure 5 shows this type of transactions, where the NCAP sends a request command and the WTIM returns, if required, a reply. 


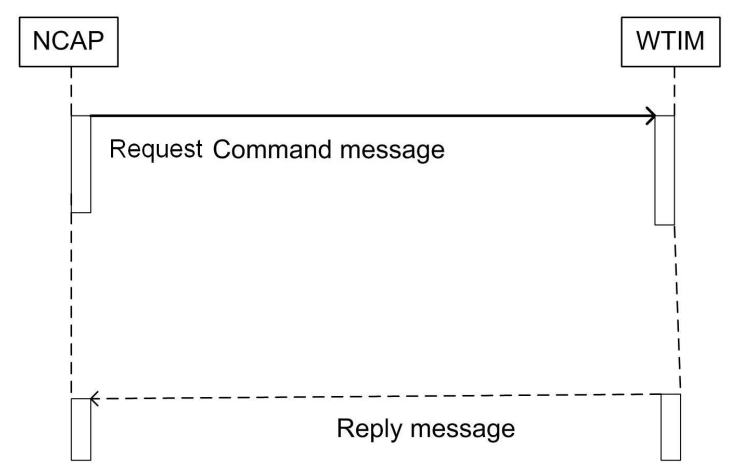

Fig 5. Transaction initiated by the NCAP.

Figure 6 shows the compliant IEEE1451.0 message structure of both the request command and the reply.

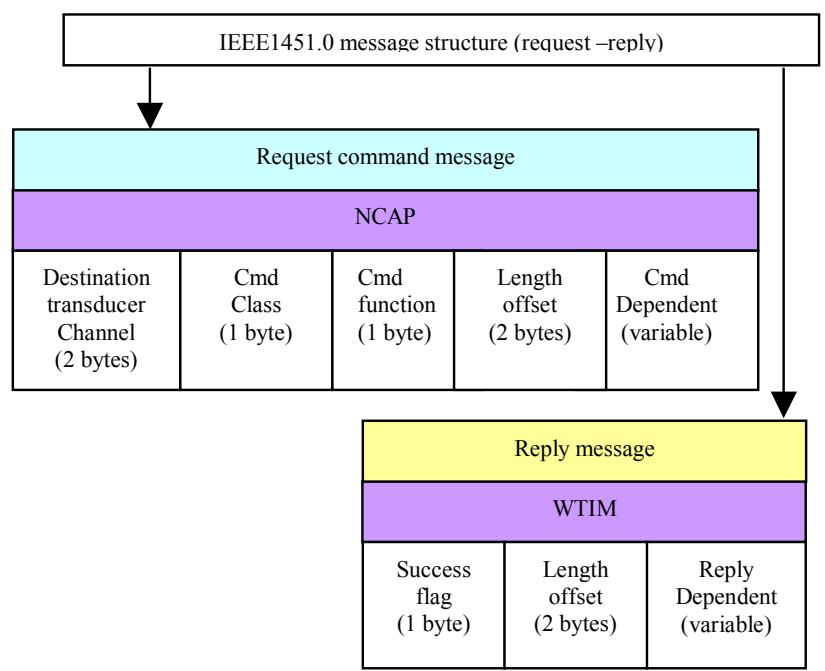

Fig. 6. IEEE1451.0 compliant message structure

The request command message from the NCAP includes:

- Destination transducer channel (2 bytes): is the maximum number of transducer channels per WTIM (65535).

- CmdClass (1 byte): defines the class of command (see Table 1).

- Cmdfunction (1 byte): defines the function of the IEEE1451 command (see table 2).

- Length offset ( 2 bytes): defines the number of bytes present in the next field.

- Cmd dependent (variable): contains additional information or data that depends on the specific command.

The reply message (WTIM response) includes:

- Success flag (1 byte): states if the command succeded or failed.

- Length offset ( 2 bytes): number of bytes present in the next field
- Reply dependent: contains additional data that depends on the specific command.

\section{A. IEEE1451.0 commands}

The IEEE1451.0 defines eight standard command classes (0-7), some reserved classes (8-127), and open classes to manufactures (128-255). Table 1 shows the three IEEE1451.0 standard commands classes implemented in our application. The CommonCmd class is used to configure all mandatory TEDS whereas the XdcrOperate class allows reading or writing the transducer channels in each WTIM.

\begin{tabular}{cll}
\hline CmdClassID & Atribute name & \multicolumn{1}{c}{ Category } \\
\hline 1 & CommonCmd & Common commands \\
3 & XdcrOperate & Transducer operating state \\
6 & TIMActive & TIM active state commands \\
\hline
\end{tabular}

Table 1. IEEE1451.0 standard command classes implemented.

Table 2 shows the specific functions implemented within each class. Some functions need a reply (yes) of the WTIM.

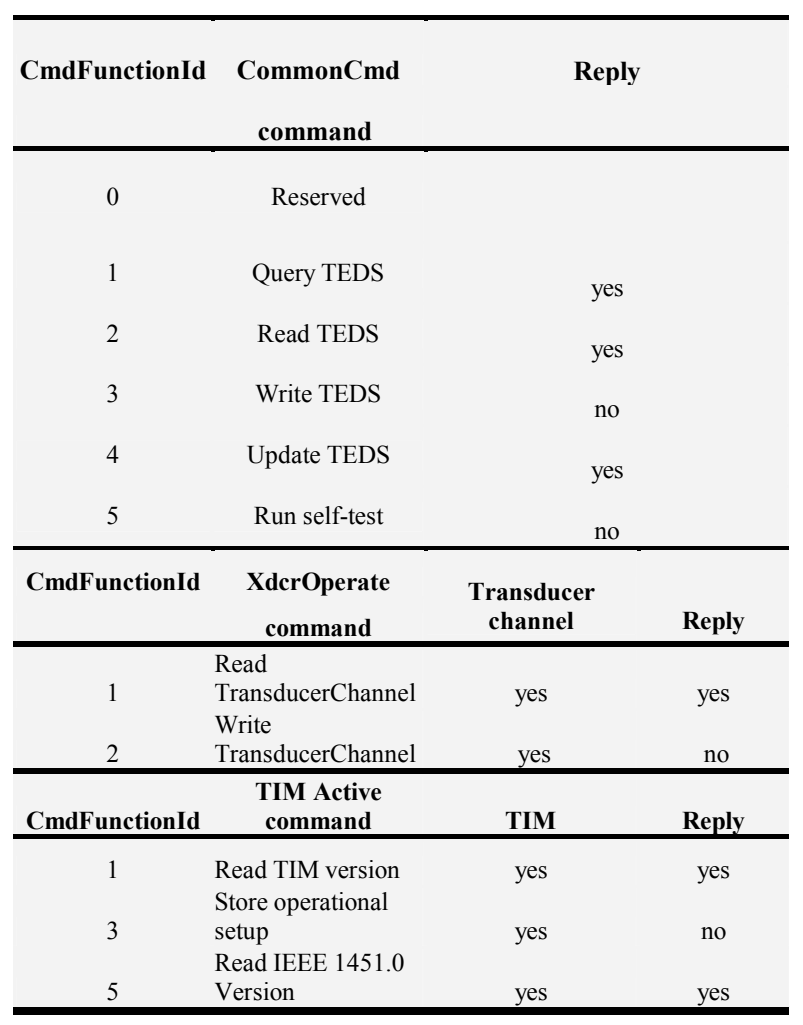

Table 2. CommonCmd, XdcrOperate, and TIM Active commands. 


\section{B. IEEE1451.5 compliant ZigBee PHY TEDS}

The concept of transducer electronic data sheet (TEDS) defines the information that requires each WTIM to operate in a WSN with IEEE1451.5 interoperability. TEDS information is stored in a non-volatile flash memory. Four mandatory TEDSs are required for each WTIM:

- Meta-TEDS: store the common information of all WTIMs.

- TransducerChannel TEDS: store the information of each Transducer Channel of the WTIM.

- User's Transducer Name TEDS: store the name by which the end user will know this WTIM.

- $\quad$ Physical TEDS: IEEE1451.5 TEDS.

Others TEDS are optional for example Frequency Response TEDS or Transfer Function TEDS. Table 3 shows the PHY TEDS implemented for the WTIMs.

\begin{tabular}{|c|c|c|c|c|c|}
\hline $\begin{array}{l}\text { Field } \\
\text { type }\end{array}$ & Field & Description & type & \# octets & Actual value \\
\hline - & & $\begin{array}{l}\text { TEDS length } \\
\text { TEDS Identification }\end{array}$ & Uint32 & 4 & $0000003 \mathrm{E}$ \\
\hline 3 & TEDSID & Header & UInt8 & 4 & 051301 \\
\hline $4-9$ & & Reserved & & & \\
\hline 10 & Radio & Radio Type & UInt8 & 1 & $2=$ ZigBee \\
\hline 11 & MaxBPS & Max data throughput & UInt32 & 4 & $250 \mathrm{Kbps}$ \\
\hline 12 & MaxCDev & Max Connected WTIMs & UInt16 & 2 & $00 \mathrm{FF}$ \\
\hline 13 & MaxRDev & Max Registered WTIMs & UInt16 & 2 & $00 \mathrm{FF}$ \\
\hline 14 & Encrypt & Encryption & UInt16 & 2 & 0000 \\
\hline 15 & Authent & Authentication & Boolean & 1 & False \\
\hline 18 & MaxSDU & Max SDU Size & UInt16 & 2 & $120200 \mathrm{FF}$ \\
\hline 19 & MinALat & Min Access Latency & UInt32 & 4 & us \\
\hline 20 & MinTLat & $\begin{array}{l}\text { Min Transmit Latency } \\
\text { Max Simultaneous }\end{array}$ & UInt32 & 4 & us \\
\hline 21 & MxXact & Transactions & UInt8 & 1 & 1 \\
\hline 22 & Battery & Device is battery powered & UInt8 & 1 & battery \\
\hline 23 & RadioVer & Radio Version \# & UInt16 & 2 & 01 \\
\hline 24 & MaxRetry & $\begin{array}{l}\text { Maximun Retries Before } \\
\text { discon }\end{array}$ & Uint16 & 2 & 00 \\
\hline 64 & Phy_Ch & $\begin{array}{l}\text { ZigBee Channels } \\
\text { supported }\end{array}$ & Uint16 & 2 & 16 \\
\hline 65 & Phy_ch_w & ZigBee channel used & Uint16 & 2 & 26 \\
\hline 66 & Phy_pwr & ZigBee Transmit power & Uint16 & 2 & $0 \mathrm{db}$ \\
\hline 67 & phyFrec & ZigBee Frecuency band & Uint16 & 2 & $2.480 \mathrm{GHz}$ \\
\hline 68 & MacChanAcc & Type Channel access & Uint16 & 2 & CSMA/CA \\
\hline 69 & BattLife & Battery life specifications & Uint16 & 2 & recharg \\
\hline 70 & Modulation & Used modulation & UInt8 & 1 & OQPSK \\
\hline 71 & RangeMax & Maxim range & Uint16 & 1 & $200 \mathrm{~m}$ \\
\hline 72 & AntennaType & Antenna type & UInt8 & 1 & integrate \\
\hline 73 & PhySensitivity & Receiver sensitivity & UInt8 & 1 & $-92 d b$ \\
\hline 74 & PhyDutyCycle & Duty cycle operation & UInt8 & 1 & $1 \%$ \\
\hline 75 & & Cheksum & UInt16 & 2 & F7 41 \\
\hline
\end{tabular}

Table 3. IEEE1451.5 compliant ZigBee PHY TEDS.
Registers (field types) 3 to 24 are common in an IEEE1451.5 WSN deployment. We have added 11 new registers for ZigBee support (registers 64-74) not included in the IEEE1451.5 standard. These registers provide useful information about the WTIM and could be included in a future revision of the IEEE1451.5 standard.

\section{RESULTS}

Tests were carried out with one NCAP and three WTIMs. We used the message structure for the NCAP request commands shown in Figure 7, where the IEEE1451.0 message (Figure 6) is encapsulated within a ZigBee IEEE1451.5 compliant frame:

- Header: includes a local group identifier (1 byte), address node ( 2 bytes), and data counter ( 2 bytes).

- ZigBee command header: includes a command type header (1 byte), packet identifier header (1 byte), sequence number ( 2 bytes) and data octects that have the correspondence with the IEEE1451.0 messages (Figure 6).

- Cycle Redundancy Check (CRC): detects possible errors in the transmission of the message.

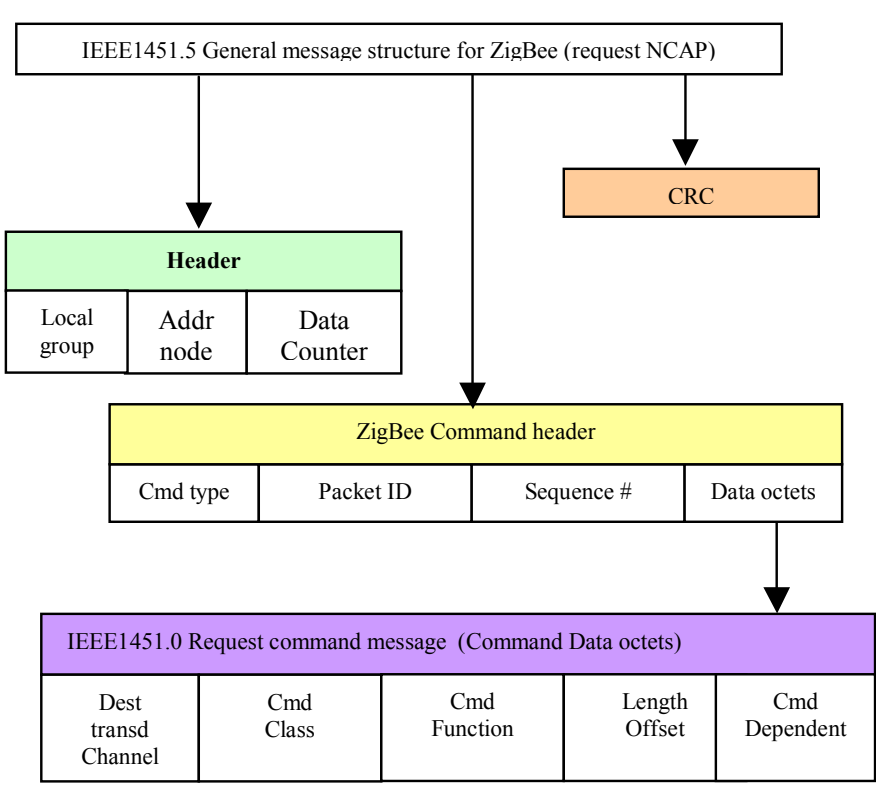

Fig. 7. General message structure for IEEE1451.5 with ZigBee interface containing a IEEE 1451.0 message (see Fig. 6)

The used steps to connect the NCAP with WTIMs were:

- Discover phase: NCAP discovers all WTIMs using the ZigBee specifications.

- Registration phase: NCAP register all WTIMs transducer channels (temperature, humidity, solar radiation, battery voltage).

- TEDS query: NCAP request information about the mandatory TEDS in each WTIM. 
- Configuration: NCAP configures the WTIMs and mandatory TEDS.

- Data exchange: Data transfer between NCAP and WTIMs to read temperature, humidity, solar radiation and battery voltage.

The NCAP node sends IEEE1451 commands and receives data information from WTIM nodes depending on the identification number (ID) in the network. Each transaction is done using an structure based in Figure 7. Each WTIM automatically performs all environmental measurements and returns the result by polled operation.

Figure 8 shows the current consumption in active mode (22 $\mathrm{mA})$ and sleep mode $(8 \mu \mathrm{A})$ for each WTIM. Expected lifetime of the batteries is more than 1 year. In the active mode the NCAP can send IEEE1451 request commands and read each WTIM transducer channel and mandatory TEDS. Each WTIM sends a data payload of 20 bytes.

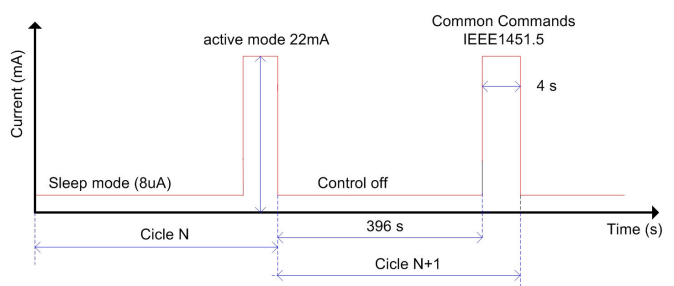

Fig 8. Operation duty cycle of a WTIM.

\section{CONCLUSION}

A ZigBee network compliant with IEEE1451 has been implemented and tested in a real deployment. The IEEE1451.0 commands have been encapsulated with IEEE1451.5 transactions for monitoring all WTIMs in active mode operation. An PHY TEDS ZigBee has been also proposed for future inclusion in the standard IEEE1451.5.

All prototype nodes WTIM include a mandatory IEEE1451.0 and IEEE1451.5 TEDS allowing interoperability and support plug and play capabilities to self-diagnosis, node calibration in environmental monitoring applications.

This deployment with IEEE1451.5 support, explores the new capabilities of smart sensor nodes in WSN with ZigBee interface using only IEEE1451.5 request - response transactions compatible with IEEE1451.0 standard.

\section{ACKNOWLEDGMENT}

This work is partially supported by the Spanish Ministry of Education and Science under contract TEC2007-66331 and by the European Regional Development Fund. Jorge Higuera is supported by the Programme Alban, the European Union
Programme of High Level Scholarships for Latin America, scholarship No. E07D400433CO.

\section{REFERENCES}

[1] IEEE 802.15.4-2006 Standard for Information technologyTelecommunications and information exchange between systems- Local and metropolitan area networks- Specific requirements Part 15.4: Wireless Medium Access Control (MAC) and Physical Layer (PHY) Specifications for Low-Rate Wireless Personal Area Networks (WPANs). IEEE Computer Society. Revision of IEEE Std 802.15.42003). ISBN 0-7381-4996-9. 8 September 2006

[2] National Institute of standards and technology. Standard IEEE1451. http://ieee1451.nist.gov.

[3] IEEE 1451.5 Standard for a Smart Transducer Interface for Sensors and Actuators Wireless Communication Protocols and Transducer Electronic Data Sheet (TEDS) Formats. IEEE Instrumentation and Measurement Society. ISBN: 978-0-7381-5599-9. Publication Date: Oct. 52007

[4] D. Sweetser, V. Sweetser, J Nemeth. Modular Approach to IEEE-1451.5 Wireless Sensor Development. SAS 2006 - IEEE Sensors Applications Symposium. Houston, Texas USA. February 2006.

[5] E. Song, K. Lee. An Implementation of the Proposed IEEE 1451.0 and 1451.5 Standards. IEEE Sensors and Applications Symposium SAS 2006. Houston Texas, USA, 7-9 February 2006.

[6] K. Lee, E. Song. “A Wireless Environmental Monitoring System Based on the IEEE 1451.1 Standards". Instrumentation and Measurement Technology Conference, 2006. IMTC 2006. Proceedings of the IEEE. pp.1931 - 1936. April 2006.

[7] D. Wobschall. "Networked sensor monitoring using the universal IEEE 1451 standard". IEEE Instrumentation and measurement magazine, pp.18-22. April 2008.

[8] Sentilla. "Low power wireless sensor module Sky Tmote" http://www.sentilla.com/

[9] Real time operative system (RTOS) TinyOS. http://www.tinyos.net.

[10] D. Gay, P. Levis, D. Culler, R. Behren. "The NesC Language: A Holistic Approach to Networked Embedded Systems". In Proceedings of Programming Language Design and Implementation (PLDI) 2003, June 2003.

[11] Texas Instruments. Single-Chip 2.4 GHz IEEE 802.15.4 Compliant and ZigBee(TM). http://focus.ti.com/docs/prod/folders/print/cc2420.html.

[12] J. Polastre, J. Hui, P. Levis, J. Zhao. "A unifying link abstraction for wireless sensor networks". Conference On Embedded Networked Sensor Systems. Proceedings of the 3rd international conference on Embedded networked sensor systems. pp: $76-89.2005$

[13] IEEE 1451.0 Standard for a Smart Transducer Interface for Sensors and Actuators. Common Functions, Communication Protocols, and Transducer Electronic Data Sheet (TEDS) Formats. IEEE Instrumentation and Measurement Society. ISBN: 0-7381-5597-7. Publication Date: Sept 212007. 